\section{Ciencia tomada. Estrategias frente al desfinanciamiento 2016-2019}

\begin{abstract}
Resumen: Basado en una investigación de campo, se describen las estrategias desplegadas por diversos grupos de investigación de la Universidad Nacional de San Martín en el contexto de desfinanciamiento de la ciencia y la tecnología, que tuvo lugar en Argentina entre 2016-2019. Se analiza el impacto de las políticas llevadas a cabo en ese periodo en la dinámica de investigación cotidiana de esos grupos. Se muestra cómo el trabajo científico en este contexto de desfinanciamiento, terminó "tomado" por una lógica de supervivencia para poder sostenerlo y se describen las distintas alternativas desarrolladas para este fin.
\end{abstract}

Palabras clave: Política científica; Período 2016-2019; Financiamiento de la CyT; supervivencia científica; Ajuste; Argentina.

Science taken. Strategies to face underfunding of science and technology 2016-2019

Abstract: Based on field research, the strategies deployed by various research groups of the National University of San Martín are described in the context of underfunding of science and technology, which took place in Argentina between 2016-2019. The impact of the policies carried out in that period on the daily research dynamics of these groups is analyzed. It shows how scientific work in this context of underfunding, ended up "taken" by a survival logic to be able to sustain it, and the different alternatives developed for this purpose are described.

Keywords: Scientific policy; Period 2016-2019; Underfunding of S\&T; scientific survival; Adjustment; Argentina.

\section{Ciência tomada. Estratégias contra o subfinanciamento 2016-2019}

\section{Resumo}

Com base em pesquisas de campo, as estratégias desenvolvidas por vários grupos de pesquisa da Universidade Nacional de San Martín são descritas no contexto do subfinanciamento da ciência e tecnologia, ocorrido na Argentina entre 2016-2019. Analisa-se o impacto das políticas realizadas naquele período na dinâmica cotidiana de pesquisa desses grupos. Mostra-se como o trabalho científico, neste contexto de subfinanciamento, acabou "tomado" por uma lógica de sobrevivência para poder sustentá-lo, sendo descritas as diferentes alternativas desenvolvidas para este fim.

Palavras-chave: Política científica; Período 2016-2019; Financiamento insuficiente de C\&T; sobrevivência científica; Ajustamento; Argentina.

\section{Ciencia Tecnología y Política \\ Año 4 N 7 Noviembre 2021}

María Soledad Córdoba

Doctora en Antropología Social Escuela IDAES-UNSAM

mcordoba@unsam.edu.ar

Karen Azcurra

Licenciada en Antropología Social y Cultural

Escuela IDAES-UNSAM azcurrakaren@gmail.com

Año $4 \mathrm{~N}^{\circ} 7$ Noviembre 2021 Fecha de recibido: 17/09/2021 Fecha de aprobado: 19/10/2021 https://doi.org/10.24215/26183188e063 https://revistas.un|p.edu.ar/CTyP ISSN 2618-3188

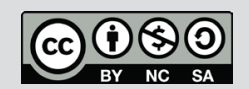

Esta obra está bajo licencia Creative Commons Atribución-NoComercial-Compartirlgual 4.0 Internacional http://creativecommons.org/licenses/bync-sa/4.O/deed.es_AR 
| Ciencia, Tecnología y Política | Año 4 | Nº7 | e063 | Noviembre 2021 | ISSN 2618-3188 | www.revistas.unlp.edu.ar/CTyP |

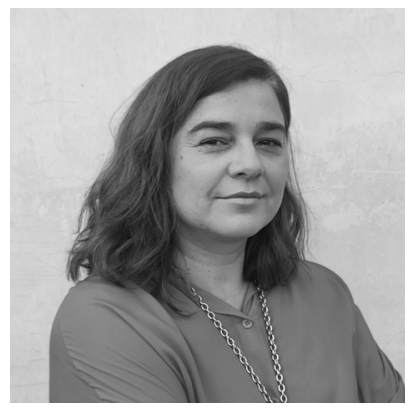

María Soledad Córdoba

Doctora en Antropología Social

Escuela IDAES-UNSAM

mcordoba@unsam.edu.ar

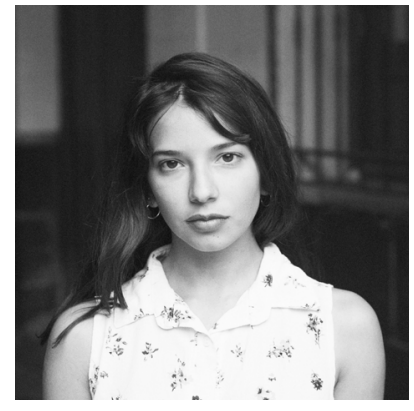

Karen Azcurra

Licenciada en Antropología

Social y Cultural

Escuela IDAES-UNSAM

azcurrakaren@gmail.com

\section{Ciencia tomada. Estrategias frente al desfinanciamiento 2016-2019}

Resumen: Basado en una
investigación de campo, se
describen las estrategias desplegadas por diversos grupos de investigación de la Universidad Nacional de San Martín en el contexto de desfinanciamiento de la ciencia y la tecnología, que tuvo lugar en Argentina entre 2016-2019. Se analiza el impacto de las políticas llevadas a cabo en ese periodo en la dinámica de investigación cotidiana de esos grupos. Se muestra cómo el trabajo científico en este contexto de desfinanciamiento, terminó “tomado" por una lógica de supervivencia para poder sostenerlo y se describen las distintas alternativas desarrolladas para este fin.

\section{Introducción}

El presente trabajo trata sobre las estrategias que desplegaron los/as investigadores/as en una universidad pública, la Universidad Nacional de San Martín (UNSAM) 1 , para sostener el quehacer científico en un contexto reconocido por los propios actores como de desfinanciamiento y escasez de recursos.

Siguiendo a Hernández (1994), la atención a los rasgos contextuales de la producción científica y a las condiciones en que la actividad de investigación se desarrolla y se comunica (se publica), remite a un posicionamiento desde la antropología de la ciencia que aquí se adoptará. Las preguntas

\footnotetext{
La UNSAM es una universidad nacional, pública y gratuita, creada en 1992. Posee un predio de cuatro hectáreas en el Partido de Gral. San Martín (Provincia de Buenos Aires), con espacios edilicios para aulas, estudio, investigación y laboratorios equipados; incrementó la matrícula de estudiantes de 837 en 2001 a 5376 en 2018 y amplió la oferta académica a 144 carreras (73 de posgrado, 61 de grado y 10 de pregrado). Desde sus inicios, ha mostrado una marcada orientación hacia la investigación realizando convenios con la Comisión Nacional de Energía Atómica (CNEA) y con el Instituto Nacional de Tecnología Industrial (INTI). Más del 65\% de sus recursos se destinan a las áreas de la CyT, con 938 personas dedicadas a investigación, desarrollo e innovación tecnocientífica (UNSAM, 2018).
} 


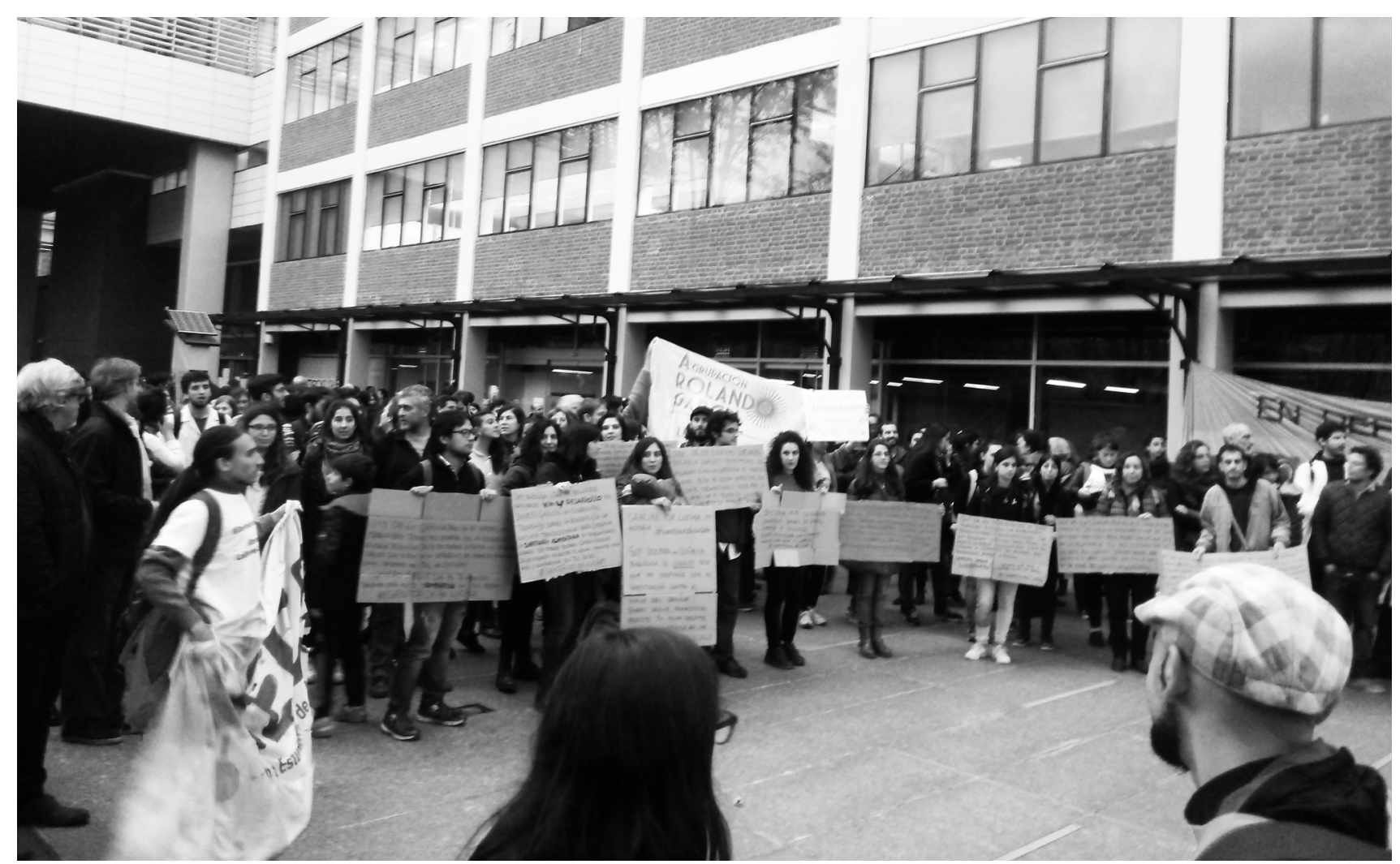

Foto 1. Movilización y reclamo en la explanada del CONICET, 22 de mayo 2019 (Foto de Karen Azcurra)

que guiarán el trabajo son: ¿cuáles han sido los impactos que ha producido la política científica implementada entre 2016-2019 en la actividad de investigación que se llevó a cabo en la universidad analizada? y ¿cuáles han sido las estrategias generadas por los/as investigadores/as para dar continuidad a su trabajo?

El punto de partida de esta investigación está constituido por una etnografía realizada en el Laboratorio de Biomateriales de la UNSAM entre 2017 y 2019, que puso en evidencia cómo el quehacer de la investigación fue cambiando significativamente como consecuencia de la política científica nacional puesta en marcha desde 2016. Sus resultados mostraron dificultades para sostener las tareas de rutina por falta de insumos, estancamiento de líneas de investigación, la imposibilidad de comprar nuevos equipos o repararlos, y la pérdida de parte del personal de investigación con que se contaba (Azcurra, 2020).
Estos resultados puntuales llevaron a extender la investigación, para analizar el impacto que estas políticas tuvieron en otros grupos de investigación que se encuentran en el Campus Miguelete de la UNSAM. Para ello, se relevó la situación de 30 centros de investigación (sobre un total de 97) de las siete unidades académicas que componen la universidad, a través de cuestionarios estructurados y entrevistas semiestructuradas. Se incluyeron equipos del área de las Ciencias Sociales y las Humanidades (CSyH) y del área de las Ciencias Experimentales y la Tecnología (CEyT). En su gran mayoría, los interlocutores fueron investigadores/ as con un rol de dirección (jefes/as de laboratorio o directores/as de centros de investigación).

\section{La retracción de la inversión estatal en ciencia y técnica (2016-2019)}

A partir del 2016, observamos una serie de medidas en la política científica nacional que afecta- 
ron y movilizaron al sector científico tecnológico argentino. Entre ellas, el achicamiento del presupuesto nacional destinado a CyT y la reducción de ingresos a carrera de investigador científico $(\mathrm{CIC})$ del CONICET. El rechazo y el descontento ante estas medidas fueron visibilizados en movilizaciones y reclamos públicos llevados a cabo por integrantes del sector que, en numerosas oportunidades, tomaron la vía pública e incluso el edificio del Ministerio de Ciencia, Tecnología e Innovación Productiva, que fue degradado a Secretaría durante el transcurso del período considerado.

Informes elaborados por actores del sector muestran, por ejemplo, que en 2019 ingresaron a la CIC del CONICET solo el 17\% de los doctores que se habían presentado en el 2018, quedando 2100 candidatos fuera (Aliaga, 2019a). Asimismo, ponen en evidencia la disminución de los montos de los subsidios para Proyectos de Investigación Científica y Tecnológica (PICT) -una de las principales fuentes de financiamiento público de la investigación en Argentina desde mediados de los noventa-, pasando de 27.000 dólares anuales en 2015, a 21.000 en 2016, continuando la reducción a 19.600 en 2017 y cayendo finalmente a 14.000 dólares anuales en 2018 (SOS Ciencia, 2018).

De manera general, "se suspendió todo intento de impulsar el desarrollo de la industria tecnológica nacional desde el Estado", se anularon convenios firmados entre ministerios del Estado $\mathrm{Na}-$ cional y empresas tecnológicas como INVAP y se suspendieron proyectos de base científico-tecnológica de relevancia estratégica como la construcción del satélite ARSAT 3 y el desarrollo del Sistema Aéreo Robótico Argentino (SARA) (Aliaga, 2019b, p. 4).

A esta retracción en el financiamiento público de la ciencia, se le sumó el retraso en el desembolso de los subsidios a la investigación ya concursa- dos y aprobados con anterioridad a 2016. Esta situación de retrasos e interrupción de pagos se vio agravada como consecuencia de la devaluación del peso frente al dólar, aspecto de vital relevancia para las actividades de investigación que requieren de insumos importados.

Organismos autárquicos como la Comisión Nacional de Energía Atómica (CNEA), el Instituto Nacional de Tecnología Agropecuaria (INTA) y el Instituto Nacional de Tecnología Industrial (INTI), reclamaron por la baja de sus presupuestos y el despido o la no renovación de contratos de investigadores/as y personal técnico. Un informe elaborado por el Centro de Investigación y Formación de la República Argentina (CIFRA) muestra que en el periodo de 2015-2019 la reducción porcentual de las partidas de la Función CyT en la CNEA fue del 36\%; en el INTA del 43\% y en el INTI del 46,2\% (Barrera, 2019).

\section{Efecto de las políticas CyT aplicadas entre 2016 y 2109 en los centros de investigación de la UNSAM}

\section{Impacto en los presupuestos de investigación}

En el Campus Miguelete de la UNSAM, las actividades de investigación están fuertemente sostenidas con financiamiento público y, en menor medida o con menor peso, con capitales privados (empresas, ONGs, etc.) y actores internacionales (proyectos o redes científicas internacionales). La financiación estatal comprende tanto los subsidios para proyectos concretos (a través de distintos fondos, la Agencia I+D+i y/o secretarías públicas) como los salarios de los/las investigadores/as o becarios/as de investigación (a través de CONICET y en menor medida de la misma Universidad).

La percepción de los/as investigadores/as con- 
sultados sobre la variación del presupuesto "en términos reales" (es decir, considerando la inflación y la devaluación del peso) resultó negativa. La amplia mayoría resaltó una disminución del presupuesto de su laboratorio. Desde su perspectiva, se trató de una disminución sustancial que, en términos cuantitativos, ubican como mayor al 50\% del presupuesto con el que contaban en 2015.

Los procesos de inflación y devaluación del peso aparecen como factores causales de relevancia del achicamiento presupuestario. Sobre este punto, uno de los entrevistados explicaba que, aun realizando una previsión de la inflación en el momento de planificar y diseñar el presupuesto de investigación, no se lograba cubrir los incrementos de los gastos que efectivamente ocurrían durante el desarrollo del proyecto (Investigador en $\mathrm{CSyH}$, comunicación personal, 30 de septiembre de 2019).

Por otro lado, el retraso del pago de los gastos rendidos y/o de los adelantos de los subsidios, también aparece como una causa importante de la disminución presupuestaria de los laboratorios y centros, dado que, en el momento de contar efectivamente con el dinero, el monto no alcanzaba para ejecutar el proyecto debido al proceso inflacionario o de devaluación ocurrido en el interín. La discontinuidad de los pagos de los subsidios (entre mediados de 2018 y agosto de 2019) y la interrupción de la comunicación con los actores estatales (específicamente CONICET y Agencia I+D+i), quienes "no respondían ningún mensaje", generó una situación de "incertidumbre e imprevisibilidad absoluta" (Investigador en $\mathrm{CSyH}$, comunicación personal, 30 de septiembre de 2019).
Sin embargo, separan este hecho de la reducción nominal de los montos de subsidios del CONICET (por ejemplo, Proyectos de Investigación Orientada y Proyectos de Investigación Plurianuales), lo cual aparece ligado a un proceso que se venía dando desde antes de 2016.

\section{Impacto en la comunicación de la ciencia, recursos y mantenimiento de laboratorios}

La mayor dificultad que se registró entre los/as investigadores/as fue en la capacidad de organizar reuniones científicas y de asistir a las mismas, ya que esta actividad es considerada parte fundamental del quehacer científico y percibida como necesaria para "presentar lo que se hace y para mirar qué hace el mundo" (Investigadora en CEyT, comunicación personal, 13 de mayo de 2019).

En este sentido, una investigadora en $\mathrm{CSyH}$ afirmaba que la investigación "requiere movilidad (...) diálogo con otras comunidades académicas que están en diferentes países y no disponer de financiamiento para eso es un problema" (Comunicación personal, 15 de julio de 2019).

Otra dificultad importante registrada fue la capacidad de reparar o comprar equipos, seguida por el problema de la adquisición de insumos importados y realizar trabajo de campo fuera del área de residencia de los/as investigadores/as.

Por último, la comunicación, difusión y divulgación de la ciencia también se vio afectada. Se registró dificultad para publicar (especialmente los equipos del área de las CEYT a causa del costo en dólares del publication fee²) y para comunicar los resultados de las investigaciones en acciones hacia la comunidad como la organización de talleres

\footnotetext{
2 El publication fee (este fue el término utilizado por los actores entrevistados) refiere a la tarifa de publicación que aplican la casi totalidad de las revistas de alto impacto en ciencias experimentales y naturales, a los autores o a las instituciones de pertenencia de los mismos. Esta tasa también se conoce como Article Processing Charge (APC) y su aplicación se justifica por ofrecer acceso abierto a los artículos, acceso mixto o híbrido (abierto/cerrado) y a los fines de costear el proceso editorial, en términos de recursos humanos e infraestructura de la revista.
} 
| Ciencia, Tecnología y Política | Año 4 | Nº7 | e063 | Noviembre 2021 | ISSN 2618-3188 | www.revistas.un|p.edu.ar/CTyP |

y otras actividades de transferencia y divulgación científica.

En las instancias de entrevistas individuales, los/ as investigadores/as señalaron una diferencia entre las necesidades de la rutina de investigación en CEyT respecto a las CSyH: mientras que en Ios equipos de CEyT la imagen fuerte y "triste", en términos de una entrevistada, era la de una "heladera que se va vaciando" (Comunicación personal, 15 de julio de 2019); en los equipos de CSyH, los investigadores bajaban sus expectativas sobre los resultados esperados, en particular si los proyectos requerían trabajos de campo fuera del área de residencia de los investigadores.

\section{Impacto en los equipos de trabajo}

Respecto al impacto sobre la incorporación de becarios/as e investigadores/as a los laboratorios y centros de investigación, no se evidenció un impacto cuantitativo importante en el número de integrantes de los equipos. Sin embargo, los/ as entrevistados/as nos señalaban que algunos de los/as becarios/as que no habían ingresado a carrera $(\mathrm{ClC})$, permanecían trabajando en el mismo equipo, aunque financiados por otras fuentes o reduciendo su jornada laboral. En todo caso, reconocen una situación de "cuello de botella" en la que, becarios/as que habían cumplido con los requisitos para ingresar a $\mathrm{CIC}$ (incluso evaluados "con el máximo puntaje"), veían truncada esa posibilidad por la reducción del número de ingresos $y$ "aunque han hecho todos los deberes con excelente, no consiguen entrar" (Investigador en $\mathrm{CSyH}$, comunicación personal, 30 de septiembre de 2019).

Por otra parte, los entrevistados del área de CEyT han reconocido que tuvieron que restringir el número de nuevos tesistas en sus equipos por no poder garantizarles el desarrollo de su investigación doctoral o posdoctoral (por falta de insumos, equipos o dinero para viáticos, etc.). A su vez, la situación de precariedad salarial en el sector CyT y la incertidumbre que generaron las medidas de la política científica mencionadas en el primer apartado, operó desalentando la incorporación de nuevos integrantes a los equipos.

\section{Estrategias frente al desfinanciamiento}

Ante la situación expuesta en los apartados precedentes, se planteó la pregunta acerca de cuáles fueron las estrategias puestas en juego por los/as investigadores/as para sostener su rutina y sus proyectos, y qué características específicas adquirió el modo de hacer ciencia en este contexto de escasez de recursos. A continuación, se detallan las estrategias más empleadas por los/ as interlocutores/as, que según ellos/as mismos, no se limitan exclusivamente la respuesta a la política científica nacional entre 2016 y 2019, sino que constituyen un modo bastante recurrente de llevar adelante la actividad científico-tecnológica en Argentina, donde la disponibilidad de recursos y financiamiento aparece más como una excepción que como la regla.

De acuerdo a las respuestas al cuestionario estructurado con el que se trabajó, la estrategia que aparece como la más utilizada por los/as encuestados/as, resultó ser el desembolso de fondos propios (autofinanciamiento) o el uso de equipamiento personal (como una computadora o equipos de baja complejidad) para sostener parte de las actividades de investigación. Los resultados mostraron que 4 de cada 10 investigadores/as entrevistados ponían en juego sus propios salarios o su capacidad de endeudamiento (a través de sus propias tarjetas de crédito), financiando viáticos, pequeñas compras de materiales, insumos importados o la publicación de papers en revistas científicas. En cuanto a los gastos de publica- 
| Ciencia, Tecnología y Política | Año 4 | N07 | e063 | Noviembre 2021 | ISSN 2618-3188 | www.revistas.un|p.edu.ar/CTyP |

ción, para las ciencias experimentales las revistas científicas de alto impacto tienen un costo que puede variar entre los 1.000 y los 4.000 dólares, de acuerdo a los testimonios de los/as entrevistados/as. Considerando la devaluación del peso, el costo de una parte importante de la actividad científica, como es la publicación de resultados, encuentra dificultades mayores para sostenerse. Según sus propios relatos, para costear los publication fees, "juntan la plata entre los/as autores" y piden "una reducción del fee, explicando al editor de la revista la situación de la ciencia en Argentina" (Investigadora en CEyT, comunicación personal, 15 de julio de 2019).

Otra modalidad de autofinanciamiento señalada fue el propio endeudamiento a través del uso de la tarjeta de crédito, lo cual implicó el riesgo de costear los intereses ante retrasos en los desembolsos. Los/as investigadores/as que optaron por esta modalidad para no frenar la actividad del laboratorio, quedaron endeudados durante 2018, año en que los pagos se atrasaron entre 9 y más de 12 meses.

Esta situación debe ser considerada en relación con la "disminución real" de los salarios de los investigadores/as, tal como lo señalaron en las entrevistas. Es decir, no sólo sus salarios cayeron por debajo de la inflación, sino que además utilizaron parte de ese salario para sostener gastos de rutina de la investigación.

Otras estrategias implicaron decisiones más radicales como la de interrumpir o suspender líneas de investigación. También manifestaron abocarse a la búsqueda y obtención de subsidios internacionales como estrategia alternativa. Algunos testimonios acordaron en que "tener financiamiento en dólares o en euros es el único recurso que queda para poder seguir manteniendo el laboratorio" (Investigadora en CEyT, comunicación personal, 11 de noviembre de 2019).

Por otra parte, una práctica de relevancia que puso en evidencia esta investigación fueron las relaciones de colaboración y "solidaridad" que establecían los/as investigadores/as del área de las CEyT entre colegas de la misma universidad o de otras instituciones como estrategia de sostenimiento de la actividad científica. De acuerdo a sus propios testimonios, recurrieron a sus colegas con frecuencia para "hacer trueques" de insumos, pedir préstamos o utilizar un equipo, instrumental o incluso capacidades cognitivas de otro laboratorio (Investigadora en CEyT, comunicación personal, 15 de julio de 2019). Estas "prácticas solidarias" sostuvieron una parte importante de la rutina, tanto por la circulación que generaron los préstamos recíprocos, como por la apertura del propio espacio de trabajo y sus recursos.

Otra experiencia sumamente significativa, relevada de las prácticas sociales de los actores, fue la circulación de información y solicitudes a través de canales de comunicación informales, como grupos de WhatsApp de egresados de carreras universitarias o de redes temáticas de investigación. De esta manera, parte de la rutina de la investigación científica fue posible de ser llevada a cabo por la activación de las redes de sociabilidad que involucraron las trayectorias de los investigadores, dando lugar a un flujo de intercambios informales de objetos e información y, más raramente, a intercambios formales a través de convenios institucionales de trabajo (Azcurra, 2020, p.75).

Por último, otro tipo de estrategia apuntó a paliar los impactos de la inflación y la devaluación del peso. Por ejemplo, para los proyectos PICT, se derivaron fondos de rubros menos prioritarios para la investigación, a los fines de elevar el monto en los rubros cruciales (operación permitida por el reglamento de rendición de los proyectos, hasta un 
tope porcentual sobre el total). En segundo lugar, para los tipos de subsidio que incluían adelantos anuales de fondos, la estrategia consistió en dolarizar los montos recibidos hasta el momento preciso de efectuar y tener que rendir un gasto. De esta manera, los/as investigadores/as lograron mantener el "valor real" del subsidio ante subas de inflación o devaluación del peso. Estas estrategias resultaron generalizadas para los equipos en ambas áreas de conocimiento y, en sus términos, permitieron "salvar los proyectos".

\section{Conclusiones}

A partir de lo expuesto, podemos identificar algunos aspectos que se destacan del quehacer científico en los laboratorios y centros de investigación de una universidad pública, durante un período que, desde la perspectiva de los actores, es considerado como de "vaciamiento" y "desfinanciamiento" de la actividad científico-tecnológica.

Respecto a las fuentes de financiamiento, observamos que el Estado se confirma como el actor de mayor peso en la motorización de inversiones en ciencia y tecnología y, tal como afirma Mazzucatto (2014), es el actor que asume los mayores riesgos en investigación, desarrollo e innovación de tecnología. Sin una intervención fuerte del Estado, "la convergencia, la 'comunicación' entre los diferentes grupos con intereses en el desarrollo de la ciencia, de la tecnología y de las ciencias del desarrollo, no será efectivo para los países periféricos" (Vessuri, 1983, p. 68).

En este sentido, otro período emblemático de retracción del Estado y referido sistemáticamente por nuestros/as interlocutores/as corresponde a los años noventa. Sin embargo, observamos que incluso en esa década de marcadas políticas neoliberales que impactaron en la reducción de fondos al CONICET e incluso en su intervención (DN 1660/96), la política científica nacional apuntó a crear nuevas fuentes de financiamiento de la investigación. Nos referimos especialmente al Banco Interamericano de Desarrollo (BID), cuyos préstamos constituyeron la base de la creación de la Agencia de Promoción Científica y Tecnología en 1996, a partir de la cual se canalizaron las principales herramientas de financiación de la ciencia en Argentina: Ios fondos FONCYT y FONTAR (Aristimuño y Aguiar, 2015).

Los vaivenes de la economía argentina, durante el periodo considerado, impactaron directamente en los equipos de investigación. Además de la disminución real de sus presupuestos de investigación, los/as investigadores/as insistieron también en marcar la depreciación del salario y del estipendio de becarios/as. Sin duda, este es un aspecto relevante y plantea la necesidad de ser indagado ulteriormente, sobre todo porque el uso de parte del salario para financiar alguna fase del proceso de investigación resultó ser una de las principales estrategias puestas en juego para sostener la rutina científica. En este sentido, la ausencia de una política de remuneraciones adecuada en el área de CyT, "refleja poca conciencia sobre la importancia de preservar y aprovechar de manera constructiva recursos humanos valiosos, en los que la sociedad ha realizado una gran inversión" (Oteiza 1996, p. 258).

Por otra parte, respecto a la imposibilidad de organizar o asistir a reuniones científicas, la reducción presupuestaria afecta una práctica común, ligada a la producción de "capital científico" (Bourdieu, 1994), la discusión de resultados y el establecimiento de contactos y redes de colaboración en la comunidad científica (Campanario, 1999, p. 399).

Sin embargo, las diferencias en las dificultades 
| Ciencia, Tecnología y Política | Año 4 | Nº7 | e063 | Noviembre 2021 | ISSN 2618-3188 | www.revistas.un|p.edu.ar/CTyP |

concretas para ambos grupos aparecen, por el lado de las CEYT, con la necesidad de insumos dolarizados o equipos e instrumentos de elevado costo; mientras que, por el lado de las $\mathrm{CSyH}$, se evidenció la dificultad para sostener viáticos del trabajo de campo como principal consecuencia de la reducción presupuestaria que afirmaron padecer. Esto indica que las medidas de política científica impactaron fundamentalmente en la base de la producción de conocimiento, tanto para las ciencias experimentales, como para las ciencias sociales.

De esta forma, si las decisiones en materia de política científica entre 2016 y 2019 operaron como factores de impacto directo sobre la actividad de investigación, "tomando" la rutina de los laboratorios y focalizando la atención de los equipos en las estrategias para su supervivencia, los eventos económicos de la devaluación y la inflación operaron como factores de impacto indirecto profundizando la ralentización de la actividad científica. Así, la ciencia quedó "tomada" por una lógica de subsistencia que desde el discurso estatal (en particular del Ministerio degradado a Secretaría de CyT) debía servir para revitalizar la capacidad de diversificar las fuentes de financiamiento, por ejemplo, recurriendo al sector privado, y dejar de depender del Estado ${ }^{3}$. Sin embargo, de acuerdo a los intercambios que mantuvimos con los/as investigadores/as no se observó que las estrategias que pusieron en juego involucraran relaciones con el sector privado, sino que, por el contrario, tendieron a fortalecer los lazos y las redes al interior del campo científico local y en algunos (pocos) casos con el campo científico internacional. Si bien la indagación de las razones de este desinterés por el sector privado excede el objetivo del presente trabajo, en otro lugar se desarrollaron en profundidad las implicancias y los sentidos de las relaciones con dicho sector que se pusieron en juego para los/as investigadores/as durante el período estudiado y que son vivenciadas como tensionantes de su construcción identitaria en el ámbito académico (Azcurra, 2020). En definitiva, los/as investigadores/as terminaron compensando la retracción presupuestaria impulsada por la política científica 2016-2019 en parte con sus propios salarios y en parte manteniendo lo que denominamos "redes de solidaridad científica", esto es, redes de relaciones entre actores del campo científico por donde circulan insumos, equipos, instrumentos y hasta incluso fondos en préstamo, bajo la forma de intercambios solidarios.

En cuanto a la relación entre el desfinanciamiento y la pérdida de integrantes de los equipos de investigación, el trabajo arrojó resultados contrastantes. Por un lado, el número de integrantes desde una mirada estrictamente cuantitativa aparecía como estable pero, por otro, las entrevistas en profundidad dejaron en evidencia que dicha estabilidad numérica era mantenida por medio de reacomodamientos de tareas, reducción de carga horaria o sustitución de la fuente de financiamiento. En segundo lugar, el hecho de que los equipos "se mantengan", pero no se estén integrando nuevos miembros define que la "buena noticia" de la estabilidad del número de integrantes podría derivar en un vaciamiento paulatino de los equipos a medida que los/as becarios/as actuales vayan culminando su formación.

La ciencia tomada por la lógica de supervivencia logra sostenerse gracias al uso o a la circulación solidaria de equipamiento, instrumental, muestras, reactivos y conocimientos o experticias. Así,

\footnotetext{
3 Algunos elocuentes ejemplos de la promoción del vínculo con el sector privado en el marco de la retracción del financiamiento estatal de la investigación, durante el período 2016-2019: Netnews (2017) y Página 12 (2017).
} 
para compensar la precariedad del financiamiento, la ciencia se hace, activa y materialmente, en esa constelación de instituciones locales que, partiendo de cada investigador, ensambla voluntades, solidaridades y reciprocidades.

Los impactos que recogimos en este trabajo, ligados específicamente a la retracción del Estado de su rol de planificador y financiador de la investigación en CyT durante el período 2016-2019, muestran la vigencia de la relación entre las decisiones en política económica, la producción científica nacional y el proyecto de desarrollo de un país (Herrera, 1968 y 1971). Las características concretas que asume esa articulación pueden observarse en la cotidianidad del Campus Miguelete de la UNSAM, operando a través de las vicisitudes que atraviesan sus investigadores/as en sus rutinas de trabajo.

\section{Bibliografía}

Aliaga, J. (21 de julio de 2019a). Ajuste en Ciencia y Técnica en la gestión de Cambiemos. Jorge Aliaga / Página personal. http://jorgealiaga.com. $\mathrm{ar} / \mathrm{p}=3209$

Aliaga, J. (2019b). Ciencia y tecnología en la Argentina 2015-2019: panorama del ajuste neoliberal. Ciencia, Tecnología y Política, 2(3), 1-9. https:// doi.org/10.24215/26183188e024

Aristimuño, F. J.; Aguiar, D. (2015). Construcción de las políticas de ciencia y tecnología en la Argentina (1989-1999). Un análisis de la concepción de las políticas estatales. Redes, 21(40), 41-80.

Azcurra, K. (2020). ¿Sueñan los científicos con ser emprendedores? [Tesis de Licenciatura en Antropología Social y Cultural, Universidad Nacional de San Martín].

Barrera, M. (2019). Evolución de los salarios de los trabajadores del CONICET y de la Administración Pública Nacional durante la gestión de Cambiemos [Informe]. Centro de Investigación y Formación de la República Argentina [CIFRA]. http:// www.centrocifra.org.ar/docs/Salarios $\% 20 y \% 20$ CyT.pdf

Bourdieu, P. (1994). El campo científico. Redes, 1(2), 131-160.

Campanario, J. M. (1999). La ciencia que no enseñamos. Enseñanza de las ciencias, 17(3), 397-410.

Hernández, V. (1994) ¡Eureka, un paper! Producción, propiedad y autoría científica. Redes, 1(1), 145-158.

Herrera, A. (1968). Notas sobre la Ciencia y la Tecnología en el Desarrollo de las Sociedades Latinoamericanas. Revista de Estudios Internacionales, 2(1).

Herrera, A. (1971). Ciencia y política en América Latina. Siglo XXI.

Mazzucato, M. (2014). El Estado Emprendedor. RBA Libros.

Netnews (4 de septiembre de 2017). Argentina ya cuenta con una Plataforma para Financiar Proyectos Científicos y Tecnológicos. https:// netnews.com.ar/nota/1476-Argentina-ya-cuenta-con-una-Plataforma-para-Financiar-Proyectos-Cientificos-y-Tecnologicos

Oteiza, E. (1996). La evolución de la política científica: nuevos y viejos desafíos culturales para América Latina en la integración supranacional. En N. García Canclini (coord.) Culturas en globalización (pp. 245-261). CLACSO, Nueva Sociedad, CNCA - Seminario de Estudios de la Cultura.

Página 12 (22 de febrero de 2017). Barañao busca "promover la investigación en el sector privado". https://www.pagina12.com. ar/18208-baranao-busca-promover-la-investi- 
gacion-en-el-sector-privado

SOS Ciencia, 2018. Información detallada sobre la desfinanciación del sistema científico y la caída del salario de investigadores, docentes y no docentes. Facultad de Ciencias Exactas y Naturales (UBA). Recuperado el 20/07/2020 de: https:// sosciencia.net.ar/wp-content/uploads/2018/08/ CyUeE.pdf.

Universidad Nacional de San Martín [UNSAM] (2018). Informe de gestión 2018. http://unsam. edu.ar/institucional//nforme-de-Gestion-2018. pdf

Vessuri, H. (1983). El papel cambiante de la investigación científica académica en un país periférico. En E. Díaz.; Y. Texera; H. Vessuri (Comp.) La ciencia periférica. Ciencia y Sociedad en Venezuela (pp. 37-72). Monte Avila. 